\title{
Aplicação simultânea de estimulação transcraniana por corrente contínua cerebelar anódica para reabilitação do equilíbrio na ataxia cerebelar: relato de caso
}

\section{Application of add-on anodal cerebellar direct current stimulation for balance rehabilitation in cerebellar ataxia: a case report}

\author{
Mariana Sacchi Mendonça' \\ Juliana Barbosa Goulardins ${ }^{2}$ \\ Carolina de Oliveira Souza ${ }^{3}$
}

\author{
Katia Monte-Silva ${ }^{4}$ [C] \\ Clarice Tanaka ${ }^{5}$ (1)
}

\begin{abstract}
1,3,5 Universidade de São Paulo (São Paulo). São Paulo, Brasil. mariana.sacchi@hc.fm.usp.br, souzaco@ig.com.br, cltanaka@usp.br ${ }^{2}$ Autora para correspondência. Universidade de São Paulo (São Paulo); Universidade Cruzeiro do Sul (São Paulo). São Paulo, Brasil. juligoulardins@gmail.com

${ }^{4}$ Universidade Federal de Pernambuco (Recife). Pernambuco, Brasil. monte.silvakk@gmail.com
\end{abstract}

RESUMO | INTRODUÇÃO: As ataxias cerebelares são um extenso grupo de doenças que causam diversos distúrbios na marcha e no equilíbrio, e que comprometem seriamente a qualidade de vida, sem opções de tratamento eficazes. A cinesioterapia é a base de programas multifacetados que incorporam mais de um enfoque, como o treinamento de coordenação e equilíbrio. Recentemente, a estimulação transcraniana por corrente contínua (tDCS) sobre o cerebelo surgiu como uma intervenção para melhorar os distúrbios do equilíbrio. OBJETIVO: Descrever a aplicação simultânea de tDCS anódica cerebelar e cinesioterapia, em sessões múltiplas diárias para reabilitação da ataxia cerebelar. MATERIAIS E MÉTODOS: Este relato de caso incluiu um paciente do sexo masculino, de 34 anos, com história de ataxia espinocerebelar há 10 anos. Seus principais objetivos eram melhorar a marcha e o equilíbrio. Ele apresentava ataxia axial e apendicular, dificuldades na marcha e no equilíbrio. O protocolo de estimulação do cerebelo consistiu na aplicação de tDCS por 20 minutos, $2 \mathrm{~mA}$, diariamente, durante duas semanas, com ânodo posicionado sobre o ínion e cátodo sobre o músculo deltóide direito. A cinesioterapia simultânea incluiu exercícios funcionais progressivos com objetivo principal de treinamento de equilíbrio. RESULTADOS: A melhora clínica foi particularmente evidenciada por uma redução de 4 pontos na Escala para Avaliação e Graduação da Ataxia após 10 sessões, enquanto a literatura recomenda a eficácia de uma nova terapia que retardaria a progressão da ataxia em 1 ponto por ano. CONCLUSÃO: Nossos resultados sugerem que a associação entre tDCS e cinesioterapia foi eficaz neste paciente; as sessões de tDCS foram seguras e bem toleradas e podem ter desempenhado um papel na melhora nos testes funcionais. Novos estudos controlados envolvendo um número maior de pacientes são necessários para analisar os benefícios destas técnicas combinadas para maximizar a reabilitação motora nesta população.

PALAVRAS-CHAVE: Ataxias espinocerebelares. Estimulação Transcraniana por Corrente Contínua. Reabilitação. Relatos de caso.
ABSTRACT | INTRODUCTION: Cerebellar ataxias are an extensive group of diseases, which cause many disorders in gait and balance that seriously impair quality of life, and without effective treatment options. Kinesiotherapy is the basis of multifaceted programs that incorporate more than one focus, such as coordination and balance training. Recently, transcranial direct current stimulation (tDCS) over the cerebellum has emerged as an intervention to improve balance disorders. OBJECTIVE: To describe a daily multiple session's simultaneous application of anodal cerebellar tDCS to kinesiotherapy for rehabilitation in cerebellar ataxia. MATERIALS AND METHODS: This case report included a 34-year-old male patient with a 10-year history of spinocerebellar ataxia. His main goals were to improve his walking ability and balance. He presented with axial and appendicular ataxia, impaired gait, and balance. The protocol used to stimulate the cerebellum consisted of twenty-minute tDCS, $2 \mathrm{~mA}$, daily applied, over two weeks, with anode positioned over the inion and cathode over the right deltoid muscle. Simultaneous kinesiotherapy included progressive functional exercises with the main objective of balance training. RESULTS: Clinical improvement was particularly evidenced by a 4-point reduction in the Scale for the Assessment and Rating of Ataxia after ten sessions, while literature recommends efficacy of a new therapy that would retard ataxia progression by 1 point per year. CONCLUSION: Our results suggest that the association between tDCS and kinesiotherapy was effective in this patient; tDCS sessions were safe and well-tolerated and may have played a role in improving functional tests. Further controlled studies involving a larger number of patients are needed to analyze the benefits of these combined techniques to maximize motor rehabilitation in this population.

KEYWORDS: Spinocerebellar Ataxias; Transcranial Direct Current Stimulation; Rehabilitation; Case reports. 


\section{Introdução}

As ataxias cerebelares são um extenso grupo de doenças que causam diversos distúrbios na marcha e no equilíbrio, e que comprometem seriamente a qualidade de vida, geralmente relacionadas ao envolvimento cerebelar. Existem várias formas de ataxias cerebelares, incluindo causas agudas (por exemplo, acidente vascular cerebral) ou distúrbios neurodegenerativos (por exemplo, ataxias hereditárias)..$^{1.2} \mathrm{O}$ cerebelo desempenha um papel fundamental no desempenho motor como intermediário e modulador da informação cortical e periférica na aprendizagem motora, tarefas automáticas e controle postural, gerando respostas antecipatórias ou compensatórias a estímulos inesperados. $\frac{3}{}$

Até o momento, não há tratamento eficaz para ataxias hereditárias e o manejo permanece de suporte e sintomático. ${ }^{4}$ No entanto, a reabilitação é mandatória para melhorar a função, a mobilidade, a incoordenação e o equilíbrio. $\mathrm{A}$ cinesioterapia é a base de programas multifacetados que incorporam mais de um foco, como coordenação e treinamento de equilíbrio. 6.7 Os tratamentos não são capazes de modificar a condição em si, mas abordam os sinais e sintomas que se instalam nos pacientes, mantendo o máximo de independência possível dentro das funções residuais intactas. $\stackrel{\otimes}{ }$ Os exercícios terapêuticos apresentam melhora significativa dos sintomas, mas precisam ser aplicados longitudinalmente para manter a funcionalidade e a independência. 9

Nos últimos anos, a estimulação transcraniana por corrente contínua (tDCS), uma modalidade de estimulação cerebral não invasiva, surgiu como uma potencial intervenção para pacientes com distúrbios de equilíbrio, pois pode influenciar a excitabilidade do cerebelo ${ }^{10,11}$, e é um método seguro e um procedimento bem tolerado. 12 Estudos anteriores mostraram resultados promissores com o uso de tDCS cerebelar para melhorar os efeitos neurofisiológicos a longo prazo e os escores clínicos de postura, marcha e funções cinéticas em pacientes com diferentes tipos de ataxia.13,14

Além disso, acredita-se que a tDCS potencializa os resultados alcançados durante o processo de reabilitação motora e representa um método poderoso para preparar a excitabilidade cortical para uma tarefa, demanda ou estimulação motora subsequente. Assim, seu uso mútuo pode otimizar as mudanças plásticas induzidas pela prática motora, levando a ganhos clínicos mais notáveis e duradouros na reabilitação. $\frac{15}{15}$ No entanto, até onde sabemos, este é o primeiro relato de caso abordando os efeitos da tDCS combinada com cinesioterapia individualizada no equilíbrio em um adulto com ataxia cerebelar.

\section{Materiais e métodos}

\section{Descrição do caso}

Este relato de caso refere-se a um paciente do sexo masculino, 34 anos, solteiro, etnia branca, que trabalhava como repositor de máquinas de café, com diagnóstico de ataxia espinocerebelar tipo III (Doença de Machado Joseph), segundo o prontuário, diagnosticado por um neurologista. Paciente relata ausência de histórico familiar prévio e de mapeamento genético, e esses dados não foram encontrados em prontuário eletrônico. A ressonância magnética mostra atrofia cerebelar. O paciente assinou um termo de consentimento livre e esclarecido, e este estudo foi aprovado pelo Comitê de Ética local (CAAE: 93840318.6.0000.5208).

Os sintomas começaram há 10 anos, quando passou a ter dificuldades e instabilidades para correr e jogar futebol. A doença evoluiu gradativamente, com o paciente percebendo dificuldade para andar e articular palavras. Três anos depois, começou a apresentar sintomas de vertigem, quando então foi diagnosticado com a doença. Quatro anos depois, participou de um programa de reabilitação, que incluiu quatro meses de intervenção fisioterapêutica e seis meses de condicionamento físico, o que restabeleceu sua autoconfiança para sair de casa. Após esse período, ele passou a andar de forma independente, sem nenhum dispositivo de marcha. Dois anos depois, foi novamente encaminhado ao serviço de fisioterapia, devido ao aumento da instabilidade na deambulação com risco de quedas. Fez cinesioterapia individualizada por três meses, com discreta melhora das queixas, quando foi encaminhado para a Clínica de Neuromodulação. Todas as informações sobre a evolução clínica e as intervenções anteriores foram coletadas no prontuário do paciente, pois não foi possível acessar os prontuários dos serviços de saúde anteriores. 


\section{Avaliação}

O exame clínico revelou ataxia axial e apendicular, nistagmo rotatório com movimentos oculares sacádicos lentos, varredura da fala com disartria, ataxia axial e marcha com aumento da base de suporte, podendo deambular de forma independente sem uso de dispositivo auxiliar, apresentando alguma instabilidade. O paciente relatou desequilíbrio ao caminhar. Força muscular em geral (grau 5), tônus e sensibilidade superficial e profunda eram normais. O teste de índex-índex demonstrou dismetria e disdiadococinesia. O teste de Romberg também foi positivo. Ao consultar o prontuário eletrônico do paciente, nenhuma eletroneuromiografia ou outros exames foram encontrados para identificar o envolvimento de nervos periféricos. Além disso, durante o exame físico, não foram encontrados déficits sensoriais. A ressonância magnética (RM) do encéfalo mostrou atrofia cerebelar e sem outras lesões ou anormalidades (Figura 1).

Figura 1. Imagem da ressonância magnética do paciente (RM)

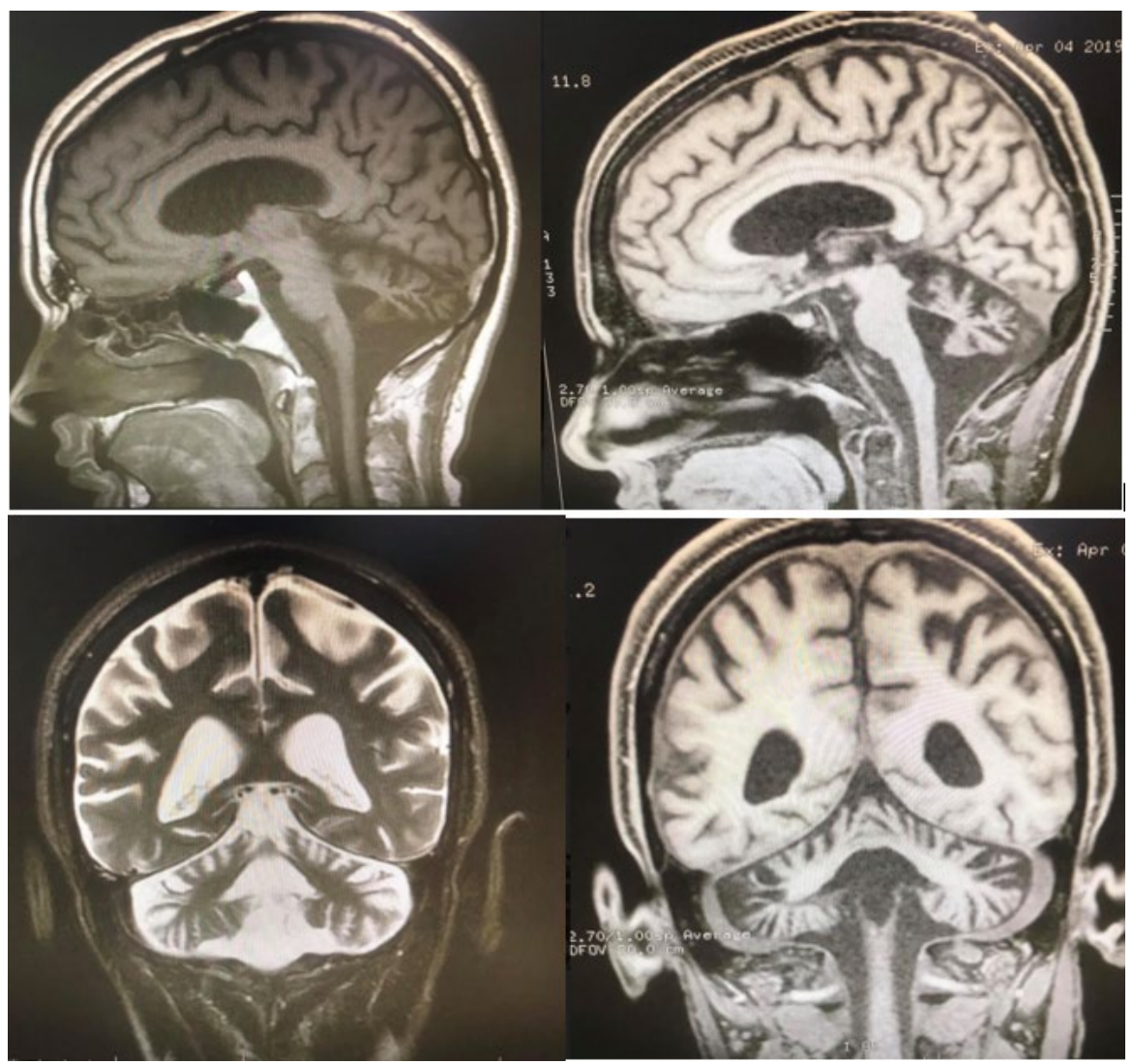

Avaliações basais da neurofisiologia cortical usando estimulação magnética transcraniana (TMS) e escalas clínicas foram realizadas, tais como: (1) Escala de Avaliação e Graduação da Ataxia (SARA), variando de 0 (sem ataxia) a 40 (ataxia mais grave); (2) Índice de Barthel (escala funcional e de mobilidade), variando de 0 a 100; e (3) Escala de Equilíbrio de Berg (BBS, preditor de risco de queda), variando de 0 a 56, todas variando da pior à melhor condição.

A SARA é uma escala subdividida em 8 itens: marcha (pontuação de 0 a 8), equilíbrio em pé (pontuação de 0 a 6 ), equilíbrio sentado (pontuação de 0 a 4), distúrbios da fala (pontuação de 0 a 6), teste de índex-índex (pontuação de 0 a 4), teste de índex- nariz (pontuação de 0 a 4), disdiadococinesia (pontuação de 0 a 4), e teste do calcanharjoelho (pontuação de 0 a 4). É realizada a soma da pontuação de cada item e, em seguida, observada a pontuação total, que pode variar de 0 (sem ataxia) a 40 (ataxia mais grave). Desta forma, a SARA é capaz de avaliar o paciente em três domínios da Classificação Internacional de Funcionalidade, incapacidade e saúde: estrutura, função e atividade. ${ }^{16}$ 
O equilíbrio foi avaliado por meio da Escala de Equilíbrio de Berg, na qual o equilíbrio do paciente é avaliado a partir de 14 atividades da vida diária que são avaliadas de 0 (pior desempenho) a 4 (melhor desempenho) pontos..$^{17}$

O Índice de Barthel é um instrumento usado mundialmente para avaliar a independência funcional e a mobilidade. Em um estudo de revisão sobre os instrumentos de avaliação em idosos, de 2004, identificou este índice como um dos mais utilizados para avaliar as atividades de vida diária. ${ }^{18} \mathrm{O}$ Índice de Barthel pertence à área de avaliação das atividades de vida diária (AVDs) e mede a independência funcional a nível de cuidados pessoais, mobilidade e locomoção. Na versão original, cada item é pontuado de acordo com a capacidade do paciente de realizar atividades de forma independente, com alguma ajuda, ou totalmente dependente de ajuda. A pontuação geral é composta pela atribuição de pontos a cada categoria, dependendo do tempo e da assistência necessária para cada paciente. Os pontos variam de 0 a 100, em intervalos de 5 pontos, e quanto maior a pontuação, mais independente são os pacientes. $\frac{19}{}$

O limiar motor hemisférico, definido como a intensidade mínima de TMS necessária para produzir uma contração visualmente detectável em 50\% dos pulsos $^{20}$, foi avaliado com o equipamento Magstim Rapid2 (Magstim, País de Gales, Reino Unido), usando uma bobina figura de 8 no córtex motor primário. Todas as avaliações foram repetidas para verificar os efeitos agudos do tratamento, duas semanas após a avaliação inicial, após a conclusão do protocolo.

\section{Intervenção}

O protocolo de estimulação do cerebelo consistiu em aplicar a tDCS por vinte minutos, com uso de $2 \mathrm{~mA}$, aplicados diariamente, exceto nos finais de semana, durante duas semanas, com ânodo posicionado sobre o ínion e cátodo sobre o músculo deltóide direito. Foi utilizado um estimulador TCT (Research Limited, Hong Kong), com eletrodos colocados em uma esponja embebidos em solução salina. Embora em muitos estudos o eletrodo estimulador, medindo 5 $\mathrm{cm} \times 5 \mathrm{~cm}$ (área $25 \mathrm{~cm} 2$ ), possa ser colocado 1-2 cm abaixo do ínion para estimulação de um hemisfério cerebelar, neste estudo optamos por usar um eletrodo estimulante maior, medindo $7 \mathrm{~cm} \times 5 \mathrm{~cm}$ (área de $35 \mathrm{~cm} 2$ ), para estimulação de todo o cerebelo. ${ }^{21}$

A cinesioterapia incluiu exercícios funcionais progressivos com o objetivo principal de treinamento de equilíbrio. O programa de exercícios incluiu: sentar e levantar; treinamento de marcha de canela, exercícios de estabilização reversa do tronco, treinamento de escadas, ajustes posturais em superfície estável progredindo para superfície instável e treinamento aeróbio com bicicleta ergométrica. Nas primeiras sessões, iniciamos com exercícios em superfície estável, com repetições e treino de estabilização (Figura 2). Os exercícios foram realizados progressivamente com a evolução do paciente. Após o tempo de ação com tDCS, continuamos os exercícios até o final da sessão. Nas sessões subsequentes, usamos superfícies instáveis para o treinamento (Figura 3). A terapia baseava-se em exercícios que enfatizavam a ativação dos músculos profundos do tronco em diferentes posturas, seguidos de exercícios de coordenação com membros superiores e inferiores, sempre com foco em exercícios funcionais, com base em uma tarefa nas sessões seguintes foi treinada marcha com tarefas que simulações de atividades funcionais e triviais, por exemplo, atravessar a rua.., $22 \mathrm{O}$ tempo total de cinesioterapia foi de cerca de 40 minutos por dia, com tDCS sendo aplicado simultaneamente durante os primeiros 20 minutos. 
Figura 2. Treino de marcha em superfície estável durante alteração de velocidade associado ao uso de tDCS anódica em região cerebelar

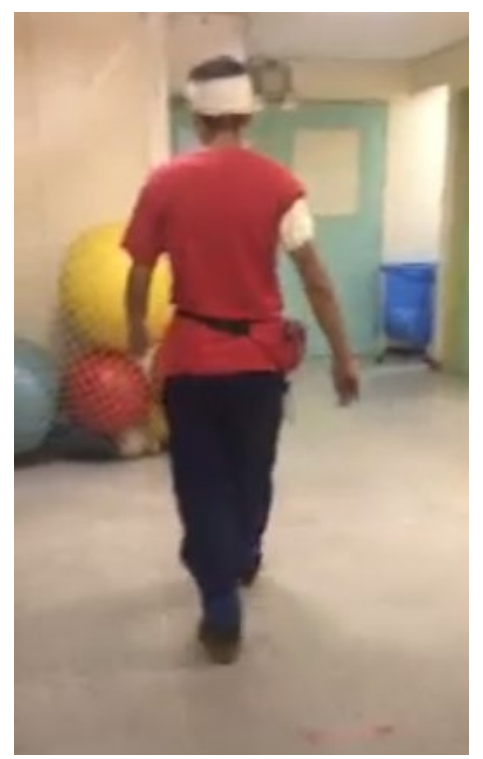

Figura 3. Treinamento de equilíbrio em uma superfície instável durante a tDCS cerebelar anódica.

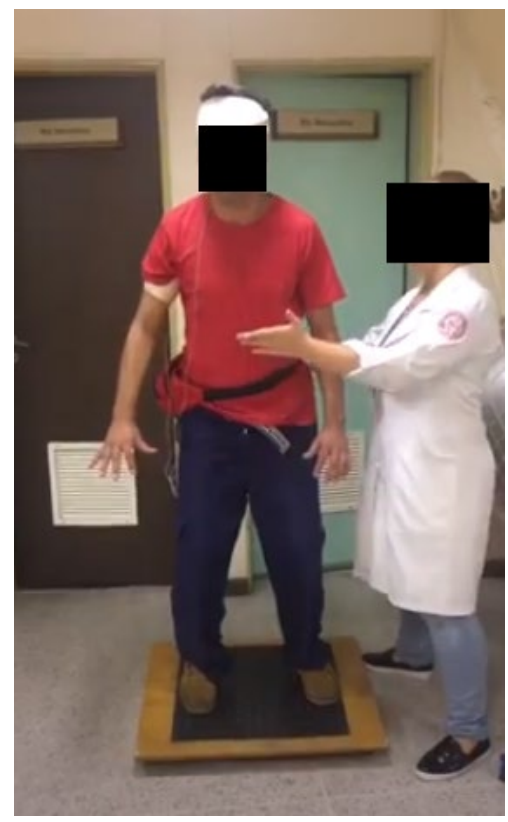




\section{Resultados}

O paciente apresentou melhora em todas as escalas e alteração do limiar motor de repouso (LMR) hemisférico, conforme a Tabela 1. Não houve relato de efeitos adversos, exceto sonolência excessiva na primeira semana de aplicação. Um questionamento sobre os efeitos adversos foi realizado diariamente antes e após as sessões.

Tabela 1. Resultados

\begin{tabular}{lcc}
\hline Avaliação & $\begin{array}{c}\text { Pré- } \\
\text { intervenção }\end{array}$ & Imediatamente após intervenção \\
\hline Escala de avaliação e Classificação da Ataxia & 12 & 8 \\
Índice de Barthel & 90 & 100 \\
Escala de Equilíbrio de Berg & 48 & 37 \\
Limiar motor hemisférico (Esquerdo/Direito) & $92 / 84$ & $84 / 78$ \\
\hline
\end{tabular}

\section{Discussão}

Nossos resultados sugerem que a associação entre tDCS e cinesioterapia foi eficaz neste paciente com ataxia espinocerebelar. As sessões de tDCS foram seguras e bem toleradas e podem ter desempenhado um papel na melhora significativa nos testes funcionais. Ao final das sessões, o paciente havia relatado uma autopercepção de bem-estar e diminuição do desequilíbrio, sendo demonstrada principalmente pela participação em festa popular de grande circulação de pessoas, cerca de três meses após o término do protocolo (conforme citou em um contato telefônico espontâneo). Isso demonstra que o tratamento atingiu não só o nível funcional das estruturas e funções do corpo, mas também as atividades e a participação social.

Foi sugerido que a estimulação anódica cerebelar produz um aumento na inibição cerebelar e, clinicamente, poderia resultar em adaptações locomotoras mais rápidas. $\stackrel{23}{\mathrm{O}}$ cerebelo desempenha um papel importante no controle da atividade motora através das conexões cerebelo-tálamo-corticais. ${ }^{24}$ As células de Purkinje exercem um tônus inibitório fisiológico sobre o córtex motor primário, denominado inibição cerebral cerebelar 25 , por meio da inibição do núcleo denteado, que tem efeito excitatório no tálamo motor ventrolateral e, eventualmente, no córtex motor. ${ }^{25,26}$ Uma diminuição do limiar motor foi observada após a tDCS combinada com a cinesioterapia neste paciente, o que representa um aumento na excitabilidade do córtex motor. Sugere-se que a estimulação cerebelar pode ter uma influência facilitadora no córtex motor, por meio de entradas excitatórias tônicas de baixa frequência que se conectam ao córtex motor do cerebelo contralateral, o que pode ser explicado pela via dento talamocortical..27

A tDCS cerebelar anódica tem sido aplicada como terapia complementar no tratamento das ataxias. É demonstrado que em pacientes saudáveis, essas técnicas são capazes de acelerar a aprendizagem motora, agindo no mecanismo de inibição do córtex motor durante os estágios iniciais de aprendizagem. Da mesma forma, nas ataxias, onde ocorre desarranjo na inibição dos núcleos cerebelares mediados por células purkinje, a estimulação cerebelar não invasiva associada a uma tarefa motora seria capaz de modular os impulsos cerebelares, restaurando a sinergia dos órgãos efetores na manutenção postura e tarefas descoordenadas, além de favorecer o aprendizado. .28

Normalmente, o tratamento de reabilitação para este perfil de paciente é consideravelmente longo, e a associação de tDCS e cinesioterapia obteve uma melhora aguda do equilíbrio em duas semanas. Pode ser devido ao efeito da tDCS como ferramenta para potencializar processos de reabilitação em um período relativamente curto, o que não foi alcançado com intervenções anteriores em adultos (apenas cinesioterapia). 
Estudos mostram que a tDCS pode modular a aprendizagem motora. ${ }^{29}$ e acredita-se que ela modifique a atividade cerebelar e altere a saída dos núcleos cerebelares. 12 Pode explicar a rápida melhora ao adicionar os estímulos em associação com o treinamento motor. A melhora clínica foi particularmente evidenciada por uma redução de 4 pontos na SARA após 10 sessões, enquanto a literatura recomenda a eficácia de uma nova terapia que retardaria a progressão da ataxia em 1 ponto por ano. ${ }^{30}$ Além disso, destacamos um possível papel da combinação de tDCS e cinesioterapia, uma vez que estudo anterior encontrou diferenças antes e após intervenção no SARA de redução de 2,8 pontos, utilizando protocolo de tDCS cerebelar anódica (5 dias / semana por 2 semanas) em vinte pacientes com ataxia. ${ }^{14}$

Outra possibilidade é que a tDCS anódica cerebelar seja capaz de aumentar a excitabilidade cortical, minimizando padrões neurológicos disfuncionais e facilitando o processo de aprendizagem em relação às reações posturais e ao equilíbrio, como visto no estudo com crianças com ataxia que realizaram tDCS anódica e treinamento em esteira. $\underline{29}$

Este relato de caso tem muitas limitações, mas muitas delas são intrínsecas aos relatos de caso. O possível efeito placebo ou efeito de confusão do avaliador não cego deve ser considerado para a interpretação deste caso. A melhora clínica pode ser atribuída à tDCS, cinesioterapia ou ambas. Estudos clínicos controlados precisam ser feitos para examinar a eficácia de cada uma dessas estratégias de intervenção separadamente e combinadas.

A pesquisa também deveria examinar o efeito dessas intervenções em pacientes com ataxia cerebelar com vários graus de dificuldade de andar e equilíbrio e cronicidade da doença. O impacto desses tratamentos em outros fatores, como incapacidade e capacidade de realizar atividades da vida diária, também deve ser examinado. Novos estudos controlados envolvendo um número maior de pacientes são permitidos para os benefícios desta técnica combinada para maximizar a reabilitação motora nesta população.

\section{Agradecimentos}

Esse estudo recebeu apoio do Conselho Nacional de Desenvolvimento Científico e Tecnológico (CNPQ - processo no. 424076/ 2018-7).

\section{Contribuições dos autores}

Monte-Silva K e Tanaka C conceberam e planejaram os experimentos. Mendonça MS, Souza CO e Goulardins JB planejaram e realizaram as simulações. Goulardins JB, Monte-Silva K e Tanaka C contribuíram na preparação da amostra e na interpretação dos resultados. Mendonça MS e Goulardins JB lideraram a redação do manuscrito. Todos os autores forneceram feedback crítico e ajudaram a moldar a pesquisa, a análise e o manuscrito.

\section{Conflitos de interesses}

Nenhum interesse financeiro, legal ou político de competição com terceiros (governo, comercial, fundação privada, etc.) foi divulgado para qualquer aspecto do trabalho submetido (incluindo, mas não se limitando $a$, doações, conselho de monitoramento de dados, desenho do estudo, preparação do manuscrito, estatística análise, etc.).

\section{Referências}

1. Marsden J, Harris C. Cerebellar ataxia: pathophysiology and rehabilitation. Clin Rehabil. 2011;25(3):195-216. https://doi. org/10.1177/0269215510382495

\begin{abstract}
2. Bultmann U, Pierscianek D, Gizewski ER, Schoch B, Fritsche $\mathrm{N}$, Timmann $\mathrm{D}$, et al. Functional recovery and rehabilitation of postural impairment and gait ataxia in patients with acute cerebellar stroke. Gait Posture. 2014;39(1):563-9. https://doi. org/10.1016/j.gaitpost.2013.09.011
\end{abstract}

3. Bodranghien F, Bastian A, Casali C, Hallett M, Louis ED, Manto $M$, et al. Consensus Paper: Revisiting the Symptoms and Signs of Cerebellar Syndrome. Cerebellum. 2016;15(3):369-91. https://doi. org/10.1007/s12311-015-0687-3

4. Fonteyn EM, Keus SH, Verstappen CC, Schöls L, de Groot IJ, van de Warrenburg BP. The effectiveness of allied health care in patients with ataxia: a systematic review. J Neurol. 2014;261(2):251-8. https://doi.org/10.1007/s00415-013-6910-6

5. Artigas NR, Ayres JS, Noll J, Peralles SRN, Borges MK, Brito CIB. Atendimento Fisioterapêutico para Indivíduos com Ataxia Espinocerebelar: Uma Revisão da Literatura. Rev Neurocienc. 2013;21(1):126-35. https://doi.org/10.34024/rnc.2013.v21.8212

6. Milne SC, Corben LA, Georgiou-Karistianis N, Delatycki MB, Yiu EM. Rehabilitation for Individuals With Genetic Degenerative Ataxia: A Systematic Review. Neurorehabil Neural Repair. 2017;31(7):609-22. https://doi.org/10.1177/1545968317712469

7. Fonteyn EM, Keus SH, Verstappen CC, van de Warrenburg BP. Physiotherapy in degenerative cerebellar ataxias: utilisation, patient satisfaction, and professional expertise. Cerebellum. 2013;12(6):841-7. https://doi.org/10.1007/s12311-013-0495-6 
8. Mitoma H, Manto M. The Era of Cerebellar Therapy.

Curr Neuropharmacol. 2019;17(1):3-6. https://doi.

org/10.2174/1570159x1701181129111212

9. Kelly G, Shanley J. Rehabilitation of ataxic gait following cerebellar lesions: Applying theory to practice. Physiother Theory Pract. 2016;32(6):430-7. https://doi.org/10.1080/09593985.2016.1 $\underline{202364}$

10. van Dun K, Manto M. Non-invasive Cerebellar Stimulation: Moving Towards Clinical Applications for Cerebellar and ExtraCerebellar Disorders. Cerebellum. 2018;17(3):259-63. https://doi. org/10.1007/s12311-017-0908-z

11. Ferrucci R, Priori A. Noninvasive stimulation. Handb Clin Neurol. 2018;155:393-405. https://doi.org/10.1016/b978-0-44464189-2.00026-3

12. França C, Andrade DC, Teixeira MJ, Galhardoni R, Silva $V$, Barbosa ER, et al. Effects of cerebellar neuromodulation in movement disorders: A systematic review. Brain Stimul. 2018;11(2):249-60. https://doi.org/10.1016/j.brs.2017.11.015

13. van Dun K, Bodranghien F, Manto M, Mariën P. Targeting the Cerebellum by Noninvasive Neurostimulation: a Review. Cerebellum. 2017;16(3):695-741. https://doi.org/10.1007/s12311016-0840-7

14. Benussi A, Dell'Era V, Cotelli MS, Turla M, Casali C, Padovani $A$, et al. Long term clinical and neurophysiological effects of cerebellar transcranial direct current stimulation in patients with neurodegenerative ataxia. Brain Stimul. 2017;10(2):242-50. https://doi.org/10.1016/j.brs.2016.11.001

15. Bolognini N, Pascual-Leone A, Fregni F. Using non-invasive brain stimulation to augment motor training-induced plasticity. J Neuroeng Rehabil. 2009;6:8. https://doi.org/10.1186/1743-0003$\underline{6-8}$

16. Schmitz-Hübsch T, Montcel ST, Baliko L, Berciano J, Boesch S, Depondt $\mathrm{C}$, et al. Scale for the assessment and rating of ataxia: development of a new clinical scale. Neurology. 2006;66(11):171720. https://doi.org/10.1212/01.wnl.0000219042.60538.92

17. Scalzo PL, Nova IC, Perracini MR, Sacramento DRC, Cardoso $\mathrm{F}$, Ferraz HB, et al. Validation of the Brazilian version of the Berg balance scale for patients with Parkinson's disease. Arq Neuropsiquiatr 2009;67(3b):831-5. https://doi.org/10.1590/S0004$\underline{282 \times 2009000500010}$

18. Paixão Júnior CM, Reichenheim ME. Uma revisão sobre instrumentos de avaliação do estado funcional do idoso. Cad Saúde Pública. 2005;21(1):7-19. https://doi.org/10.1590/S0102311X2005000100002

19. Alves-Guerreiro J. Measuring Health: A Guide to Rating Scales and Questionnaires. Physl Ther Rev. 2000;5(3):183. https://doi. org/10.1179/ptr.2000.5.3.183
20. Sandrini M, Umiltà C, Rusconi E. The use of transcranial magnetic stimulation in cognitive neuroscience: a new synthesis of methodological issues. Neurosci Biobehav Rev. 2011;35(3):51636. https://doi.org/10.1016/j.neubiorev.2010.06.005

21. Ferrucci R, Cortese F, Priori A. Cerebellar tDCS: how to do it. Cerebellum. 2015;14(1):27-30. https://doi.org/10.1007/s12311014-0599-7

22. Ilg W, Synofzik M, Brötz D, Burkard S, Giese MA, Schöls L. Intensive coordinative training improves motor performance in degenerative cerebellar disease. Neurology. 2009;73(22):1823-30. https://doi.org/10.1212/wnl.0b013e3181c33adf

23. Jayaram G, Tang B, Pallegadda R, Vasudevan EV, Celnik P, Bastian A. Modulating locomotor adaptation with cerebellar stimulation. J Neurophysiol. 2012;107(11):2950-7. https://doi. org/10.1152/jn.00645.2011

24. Holdefer RN, Miller LE, Chen LL, Houk JC. Functional connectivity between cerebellum and primary motor cortex in the awake monkey. J Neurophysiol. 2000;84(1):585-90. https://doi. org/10.1152/jn.2000.84.1.585

25. Galea JM, Jayaram G, Ajagbe L, Celnik P. Modulation of cerebellar excitability by polarity-specific noninvasive direct current stimulation. J Neurosci. 2009;29(28):9115-22. https://doi. org/10.1523/jneurosci.2184-09.2009

26. Daskalakis ZJ, Paradiso GO, Christensen BK, Fitzgerald PB, Gunraj C, Chen R. Exploring the connectivity between the cerebellum and motor cortex in humans. J Physiol. 2004;557(Pt 2):689-700. https://dx.doi.org/10.1113\%2Fjphysiol.2003.059808

27. Ugawa Y. Cerebellar Stimulation in Normal Subjects and Ataxic Patients [Internet]. In: Hallet M, Chokroverty S. Magnetic Stimulation in Clinical Neurophysiology. 2a ed. Oxford: Butterworth-Heinemann; 2005. p. 197-210. Disponível em: https://www.sciencedirect.com/book/9780750673730/magneticstimulation-in-clinical-neurophysiology

28. Benussi A, Koch G, Cotelli M, Padovani A, Borroni B. Cerebellar tDCS in patients with ataxic disorders: A double-blind, randomized, crossover, sham-controlled study. Clin Neurophysiol. 2016;127(3):e23-e24. https://doi.org/10.1016/j.clinph.2015.11.066

29. Grecco LA, Oliveira CS, Duarte NA, Lima VL, Zanon N, Fregni F. Cerebellar transcranial direct current stimulation in children with ataxic cerebral palsy: A sham-controlled, crossover, pilot study. Dev Neurorehabil. 2017;20(3):142-8. https://doi.org/10.3109/1751 8423.2016.1139639

30. Lee YC, Liao YC, Wang PS, Lee IH, Lin KP, Soong BW. Comparison of cerebellar ataxias: A three-year prospective longitudinal assessment. Mov Disord. 2011;26(11):2081-7. https:// doi.org/10.1002/mds.23809 